\title{
Courbes enveloppes des débits de crues en fonction des surfaces réceptrices
}

\author{
ESSAI DE FORMULATION ANALYTIQUE
}

\section{Overall flood discharge curves for various catchment aereas}

\author{
AN ATTEMPT AT AN ANALYTICAL FORMULATION
}

par Mue P. LARIVAILTE,

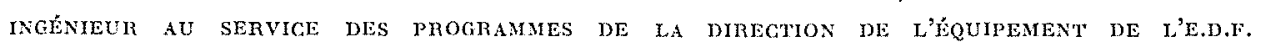

\begin{abstract}
Classement selon de grandes régions d'un nombre considérable de résultats de mesures ou d'observations relatives aux débits de divers cours d'eau à la surface da globe, et faisant l'objet d'une étude considerable de M. Pardé. Application à ces régions de courbes-enveloppe:; de deux types, que deux ou trois parametres, selon le cas, suffisent à déterminer.
\end{abstract}

C'est à la suite d'un travail considérable de M. Pardé sur les crues et à sa demande que j'ai été conduite à étudier le problème de la formulation analytique des courbes enveloppes des débits maximaux.

Ces courbes limitent supérieurement le nuage des points représentant les débits des plus fortes crues observées dans une région pour des bassins déterminés. Mais cette définition reste encore le plus souvent idéale. Les points du nuage ne peuvent être donnés avec des degrés de

\begin{abstract}
Classitication into major regions of a considerable number of observed discharge data for rivers in narious parts of the world, which Mr. Parde has considered in a very comprehensive studly.

Application of two types of overall flood discharge curve to these regions; depending on the case considered, the determination of these curves only involves two or three parameters.
\end{abstract}

probabilité constants, d'où un certain mélange. De plus, les combinaisons exceptionnelles risquent de ne pas être représentées et les enveloppes ont ainsi un tracé provisoire susceptible de déplacement vers le haut du graphique.

Néanmoins, ces courbes offrent un moyen rapide, très couramment employé, pour la prévision d'une crue et de nombreux auteurs ont tenté d'en rechercher des expressions analytiques. Diverses solutions sont exposées, en particulier dans les communications présentées au $4^{\mathrm{e}}$ Congrès des Grands Barrages (New-Delhi, 1951).

\section{FORMULES ANTÉRIEURES}

Ơn a d'abord essayé des solutions théoriques: coefficients constants, donc indépendants des régions étudiées, puis des solutions géographi- ques qui s'expriment en fonction des cadres régionaux.

La plupart sont d'ailleurs du type $\mathrm{Q}=\mathrm{A} \mathrm{S}^{n}$, 
$Q$ étant le débit maximal absolu, $S$ la surface réceptrice.

Parmi les premières, nous citerons les formules de :

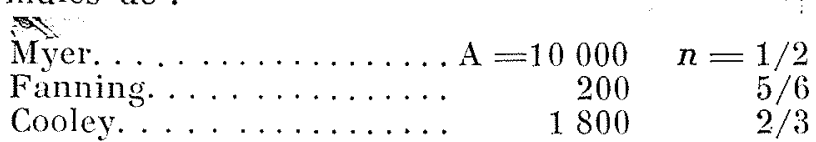

(S en miles carrés, $Q$ en pieds cubes/seconde).

L'emploi de telles formules peut conduire à des erreurs considérables, car vouloir représenter les crues maximales dans les diverses régions du monde avec un seul parametre et un exposant qui serait le même pour tout le globe est a priori contraire aux réalités géophysiques. A moins d'anomalies extraordinaires et, par exemple, de l'absorption en proportion énorme des pluies par le sol ou de la rétention lacustre dans les domaines supérieurs, la progression vers l'aval et l'accroissement correspondant de la surface réceptrice entrainent bien entendu une diminution du débit spécifique : l'intensité pluviale ne peut, en effet, ni maintenir son paroxysme sur une étendue plus vaste et basse, ni durer assez longtemps pour que tous les affluents concourrent a soutenir le flot principal. Mais pour que dans une région donnée le paramètre et l'exposant restent les mêmes quels que soient les bassins et les surfaces, il faudrait d'abord que la diminution de la tranche pluviale tombée, et plus encore de la tranche pluviale écoulée, se règle toujours d'après de mêmes taux exponentiels. Or cette uniformité du taux de décroissance pluviale a de grandes chances de ne point exister, étant données les influences variées du relief, de l'exposition, influences que l'on retrouve d'ailleurs sur la genèse de la pluie.

Ensuite, l'eau qui s'écoule ne représente qu'une fraction de toute la pluie qui tombe et en général, plus le bassin est petit et plus le quotient d'écoulement (rapport de la pluie écoulée à la pluie tombée durant l'intumescence du phénomène) a de chances d'être fort. Mais là encore, les fréquences partielles et cumulées des diverses altitudes, les natures différentes du sol, la température, l'humidité de l'air ne peuvent que faire varier, sauf par hasard, cette réduction des quotients d'écoulement en fonction des surfaces croissantes.

En outre, il se peut que, dans certains pays, aucune surface réceptrice supérieure à 30000 ou à $50000 \mathrm{~km}^{2}$ ne soit drainée par un réseau favorable aux concordances lors des grandes crues, tandis que, dans une autre région, un bassin, voire plusieurs, auront des réseaux propices aux concordances monogéniques; et les débits spécifiques records décroîtront beaucoup plus en fonction des surfaces réceptrices dans la première région que dans la seconde.

Ainsi, pour chaque région, la formule doit être différente. De très nombreux travaux ont été consacrés à l'établissement de formules régionales, toujours du type $\mathrm{Q}=\mathrm{A} \mathrm{S}^{n}$. M. Coutagne en fait un bref recensement dans sa communication au $4^{\circ}$ Congrès des Grands Barrages.

Mais encore une telle représentation peut-elle conduire à de graves erreurs; elle ne tient en effet pas toujours compte de certains phénomènes géophysiques susceptibles de se produire : aplatissement ou au contraire augmentation trop rapide des débits maximaux.

Un autre type de formules a été exploité par divers auteurs. C'est une expression à trois paramètres, de la forme:

$$
\left(\frac{A}{B+S}+C\right) S
$$

Kuichling et Murphy admettent l'un et l'autre des paramètres constants. Par contre, les Italiens Scimemi, Baratta, Pagliaro, appliquant cette formule à leurs bassins, font varier les différents paramètres suivant l'étendue des bassins et quelquefois, leurs caractéristiques géographiques. On trouve par exemple ( $\mathrm{S}$ en $\mathrm{km}^{2}$, $\mathrm{Q}$ en $\left.\mathrm{m}^{3} / \mathrm{s}\right)$ :

$$
\begin{aligned}
\mathrm{Q} & =\mathrm{S}\left(\frac{600}{\mathrm{~S}+10}+1\right) \\
& \text { pour } \mathrm{S}<1000 \mathrm{~km}^{2} \text { (Scimemi) } \\
& =\left(\frac{280}{\mathrm{~S}}+2\right) \mathrm{S} \text { (Baratta) }
\end{aligned}
$$

pour les cours d'eau des Apennins, de la Ligurie et de la Toscane. Forti introduit même un quatrième paramètre qui tient compte de l'intensité des précipitations.

Mais chacune de ces formules n'est applicable qu'à une région très réduite et il ne semble pas qu'il soit possible de la généraliser. Une brève étude mathématique signale la présence d'un point d'inflexion sur la courbe représentant en coordonnées logarithmiques la variation des débits spécifiques en fonction des surfaces réceptrices, et une augmentation sans réserve des débits bruts.

Les Australiens J. Baird et J. F. Mclllwraith, étudiant les bassins de leur pays, puis réunissant quelques données relatives à des bassins appartenant à diverses parties du monde, expriment le débit spécifique maximal $q$ en fonction de l'aire du bassin $\mathrm{S}$ par une formule du genre:

$$
q=\frac{\mathrm{A}}{(\mathrm{B}+\mathrm{S})^{n}}
$$

Cette formule laisse entrevoir l'existence du phénomène d'aplatissement des débits maximaux bruts de l'amont vers l'aval dans le cas où $n$ est supérieur à 1 ; elle voudrait donc être plus générale que les précédentes. 


\section{FORMULES PROPOSÉES}

\section{Les données}

Nous disposions de quelque 600 résultats représentant les débits maximaux des plus grandes crues connues dans le monde.

Ces résultats ont été étudiés avec minutie par M. Pardé qui, entre autres, a essayé d'en définir les degrés d'exactitude, ceux-ci s'avérant bien entendu variables d'une région à l'autre. En fait, un grand nombre des chiflres utilisés ont pu être contrôlés, rectifiés dans certains cas et M. Pardé pense qu'ils peuvent fournir des valeurs dignes de confiance à 10 ou $15 \%$ près.

D'une façon générale, il semble que l'on tende à exagérer plus ou moins les débits de crues exorbitants animés par des courants torrentiels et charriant beaucoup de matérianx plus ou moins grossiers: on augmente souvent la vitesse en sous-estimant la rugosité. Cependant, nous n'avons aucune certitude que cette erreur ait été générale et représente un pourcentage sensible des vraies valeurs.

Nous espérons donc que l'ensemble des chiffres présentés ne souffre point d'une exagération trop grave. De toutes façons, cette exagération s'appliquerait aux records observés, mais pas nécessairement aux plus grandes crues possibles dans les mêmes régions.

Ensuite, tous les débits pris en compte ne correspondent pas it des phénomènes de même fréquence. Néanmoins, nous sommes quasi certains que la probabilité d'apparition de chacum d'eux est extrêmement faible pour la plupart des cours d'eau. Presque tous les chiffres que nous domnons représentent des phénomènes d'une grande violence relative et beaucoup d'entre eux ne doivent guère pouvoir être dépassés très fortement dans l'avenir si le climat ne change pas de manière fondamentale. Aussi les tracés des courbes enveloppes des points marqués sur nos graphiques potirront être considérés sans erretir capitale, comme représentant des crues déjà fort redoutables.

Bien entendu, il n'est pas exclu qu'on doive dans un avenir plus ou moins proche repousser certains points.

$$
\text { * }
$$

Ceci dit, il n'était évidemment pas question d'étudier la courbe enveloppe, unique, pour l'ensemble de toutes les crues retenues. Nous avons donc dû distinguer celles-ci suivant certaines régions. Mais les délimitations ainsi adoptées restent encore très grossières. Pour tenter de les effectuer arec des chances de plus grande réussite, il eut fallu avoir en plus grand nombre des connaissances suffisamment approchées des débits maximaux réellement exceptionnels en certaines régions ou sous-régions. Et en chacune des zones étudiées, les crues records conformes à 15 ou $20 \%$ près aux chiffres types de M. Pardé ne sont possibles que sur certaines rivières particulièrement fougueuses. Les autres n'atteignent dans leur paroxysme que la moitié ou le tiers de ces résultats exceptionnels.

Compte tenu des superficies des bassins versants, des régimes climatiques et hydrologiques, nous avons été conduits à introduire quelques nuances. Nous distinguons par exemple l'Europe méditerranéenne, l'Europe centrale, l'Europe Grands bassins, la France... Pour les EtatsUnis, plusieurs sous-secteurs sont égalcment ćtudiés séparément.

En définitive, nous retenons 21 grandes régions et les distinctions se présentent ainsi :

Certes, on relèvera encore diverses anomalies. Celles-ci sont d'ailleurs inévitables; en effét, les délimitations régionales ou numériques sont

\begin{tabular}{|c|c|}
\hline PetrTs et Moyens bassins & Grands bassins \\
\hline $\begin{array}{l}\text { - France. } \\
\text { - Espagne (points communs avec Europe G.-B.). } \\
\text { - Italie. } \\
\text { - Europe méditerranéenne. } \\
\text { - Eurore centrale. } \\
\text { - USA - Petits bassins. } \\
\text { - USA - Moyens bassins. } \\
\text { - Texas + Nebraska. } \\
\text { - Californie du Nord. } \\
\text { - « Cyclone Diane», aout } 1955 . \\
\text { - Nouvelle-Zélande. } \\
\text { - Australie. } \\
\text { - Japon. }\end{array}$ & $\begin{array}{l}\text { - Europe, grands bassins. } \\
\text { - USA, grands bassins. } \\
\text { - Canada. } \\
\text { - Extrême-Orient, grands bassins. } \\
\text { - Afrique noire. } \\
\text { - Amérique du Sud. } \\
\text { - U.R.S.S. }\end{array}$ \\
\hline
\end{tabular}


très fuyantes et indécises vers certaines frontières.

On constatera par exemple que quelques débits indiqués par M. Pardé comme issus de bassins petits et moyens, arbitrairement limités à $20000 \mathrm{~km}^{2}$, proviennent de superficies supérieures.

Mais M. Pardé a remarqué que ces crues étaient monogéniques ou causées par une seule averse, une seule poussée hydrométrique et il a jugé que pareils phénomènes constituaient des signes distinctifs pour les rivières de bassins seulement moyens au même titre que l'étendue des surfaces réceptrices. C'est vaai surtout pour celles qui, grâce à des pentes assez vives et aux conformations concentrées de leurs réseaux, réagissent rapidement aux effets des pluies. Puis, au-delà des stations considérées, les bassins totaux de ces rivières ne deviennent pas beaucoup plus vastes qu'à l'amont desdits lieux et elles ne pourraient être comptées parmi les cours d'eau qui plus en aval auraient mérité le qualificatif de « grands ».

En outre, dans les tableaux relatifs aux grands bassins de l'U.R.S.S., puis de l'Afrique intertropicale, on inclut diverses données applicables à des bassins petits el moyens. C'est que pour les causes des crues possibles à l'issue de 20000 , $10000 \mathrm{~km}^{2}$ et moins, on manquait de données suffisamment nombreuses et précises. Pour les dites régions, il peut paraitre souhaitable de substituer par la pensée aux mots «grands bassins », «bassins de toutes étendues».

Cette relative absence de rigueur dans les distinctions entre bassins de diverses grandeurs n'est qu'apparente : leur dimension ne doit pas ètre le seul critère, des divisions trop catégoriques eussent en réalité nui à l'étude des phénomènes.

\section{Les courbes}

Pour chaque région naturelle ainsi définie, nous représenterons en coordonnées logarithmiques les différentes crues retenues en portant en abscisses les surfaces réceptrices des bassins considérés, exprimées en $\mathrm{km}^{2}$, en ordonnées les débits spécifiques correspondants en $1 / \mathrm{s} \mathrm{km}^{2}$. Puis nous tracons à vue les courbes enveloppes de ces points. La plupart de ces courbes, bombées vers le haut, offrent une décroissance régulière (fig. 1).

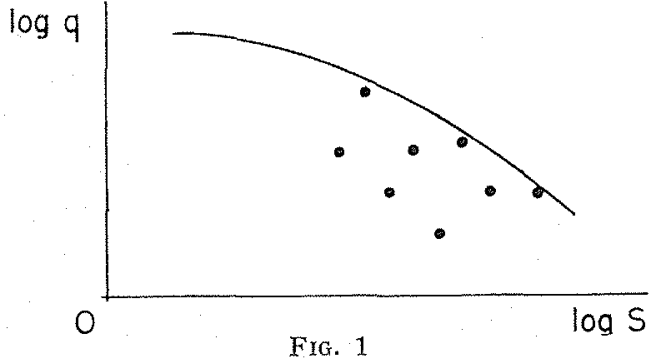

Trois se présentent avec point d'inflexion (fig. 2), c'est-à-dire que l'augmentation de la surface réceptrice n'entraine pas la même régularité de décroissance de l'abondance spécifique. Ce sont celles relatives à :

- l'U.R.S.S.,

-- l'Afrique noire,

- l'Amérique du Sud.

\section{Leur expression analytique}

Ces deux types de courbes seront étudiés séparément.

$1^{\circ}$ Pour le premier, classique, nous avons repris tout d'abord la formule présentée par les Australiens au $4^{\circ}$ Congrès des Grands Barrages :

$$
y=\frac{A}{(B+S)^{n}}
$$

qui nous semblait vouloir représenter de façon satisfaisante le phénomène de crues,

Divers ajustements ont ainsi été efrectués et les résultats obtenus seraient satisfaisants. Mais le nombre des paramètres utilisés, trois, paraît excessif; le nombre des points qui serviraient à la détermination d'une courbe enveloppe expérimentale est déjà lui-mème assez réduit.

Cherchant alors une formule à deux paramètres, nous pensons à une expression en:

$$
\ll \log -\log . »
$$

qui s'écrirait :

$$
\log \frac{q}{B}=\log \left(\log \frac{A}{S}\right)
$$

Log $=\log$. népériens, $\log =\log$. décimaux.

ou :

$$
q=\mathrm{B}\left(\log \frac{\mathrm{A}}{\mathrm{S}}\right)^{2,3}
$$

$B$ et A sont deux paramètres d'échelle que l'on estime à partir de deux points particulièrement significatifs dont l'un correspondra par

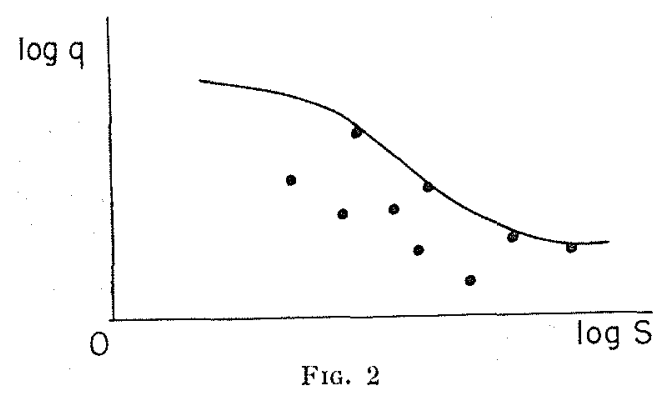

exemple à la plus grande surface réceptrice de la région considérée. On s'assure ainsi que la condition $\mathrm{S}<\mathrm{A}$ est bien vérifiée. 
La valeur de ces paramètres pour chacune des régions est consignée dans le tableau suivant:

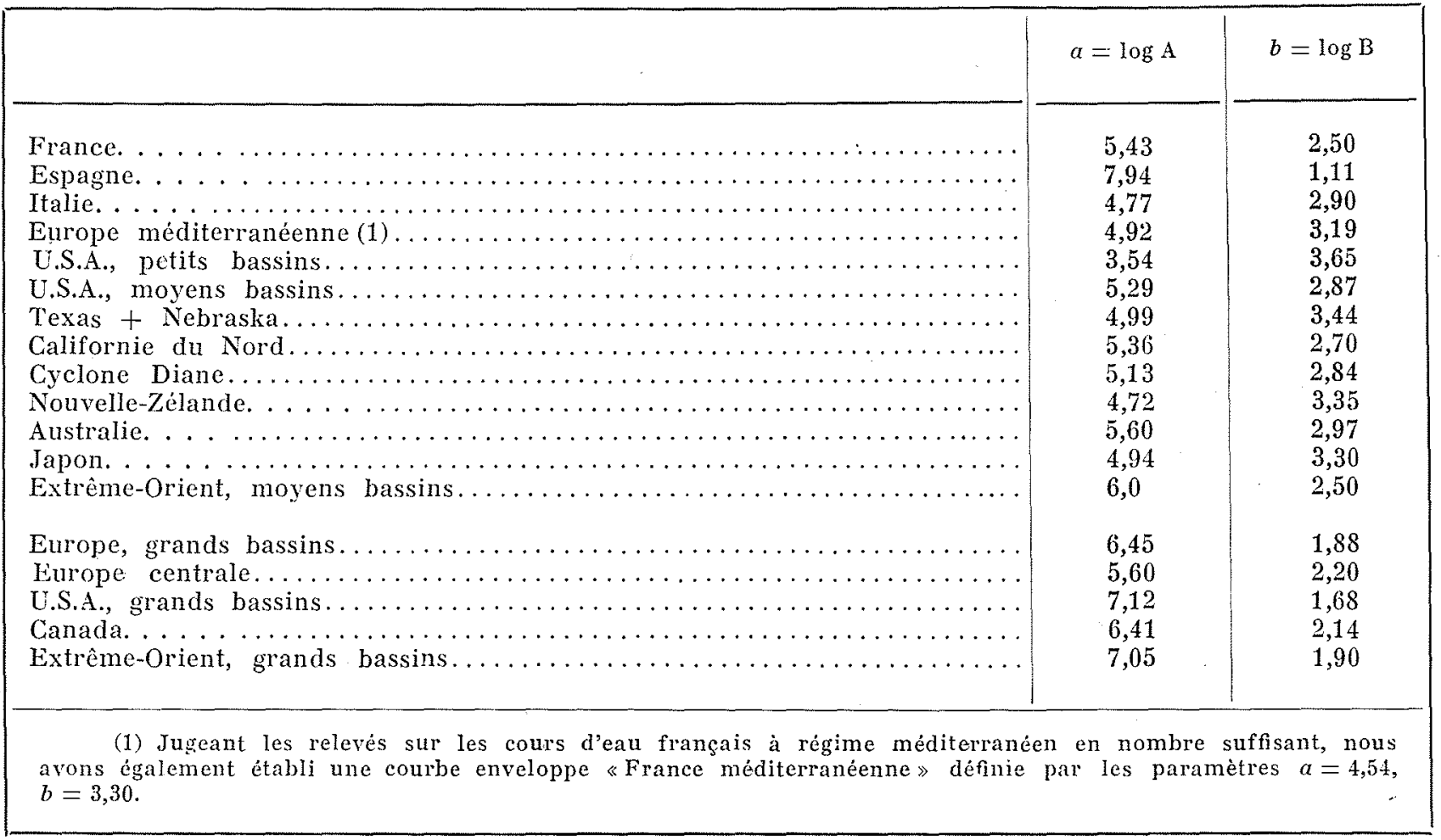

Les divers ajustements ainsi effectués (fig. 3, $a$ à e) s'avèrent, dans l'ensemble, très convenables. On trouve néanmoins encore quelques divergences, par exemple pour le Canada ou la Californie du Nord.
Mais dans le premier cas les données sont très hétérogènes; elles englobent des crues d'origine fort différentes et se produisent sur des bassins de superficies très variées, depuis 100 jusqu'à quelque un million de $\mathrm{km}^{2}$.

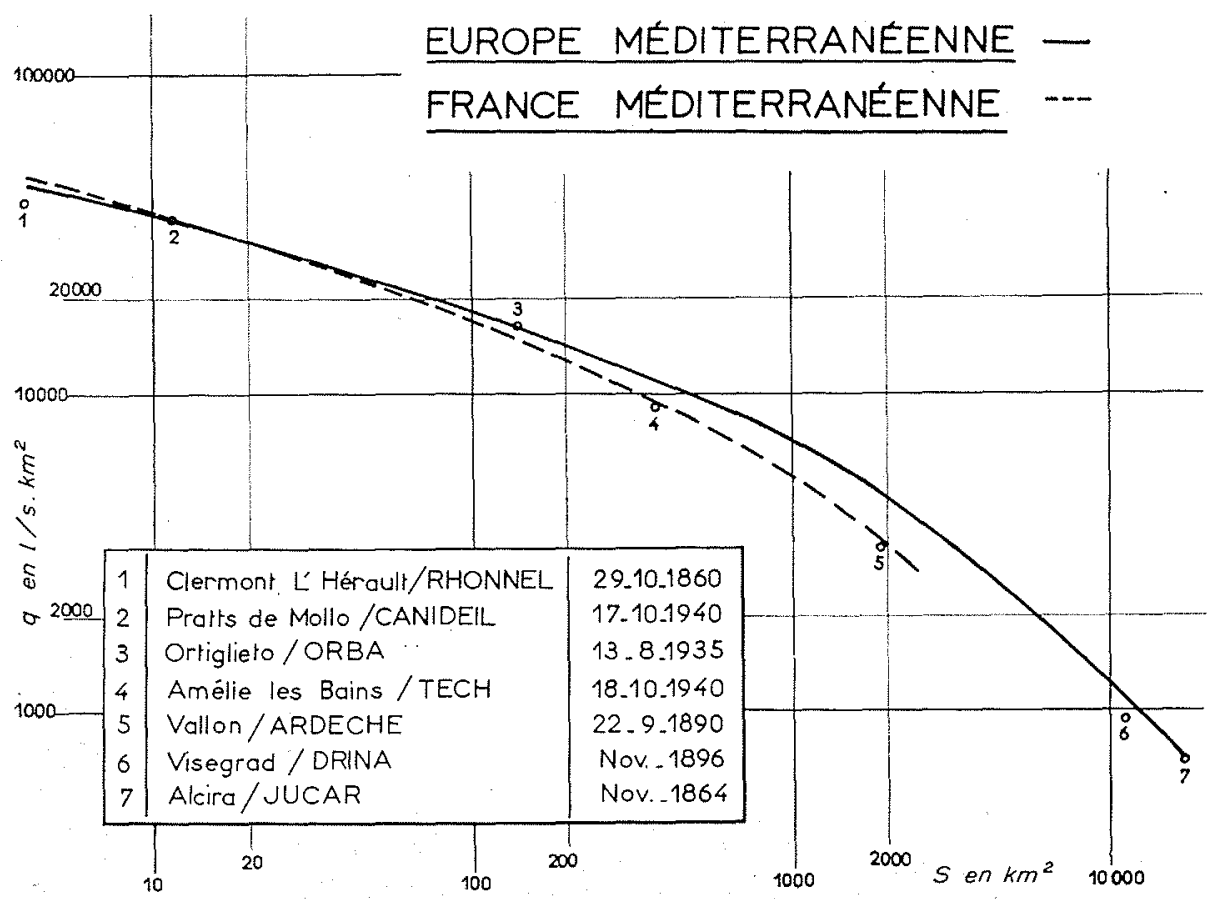

FIG. $3 a$ 
FiG. $3 b$

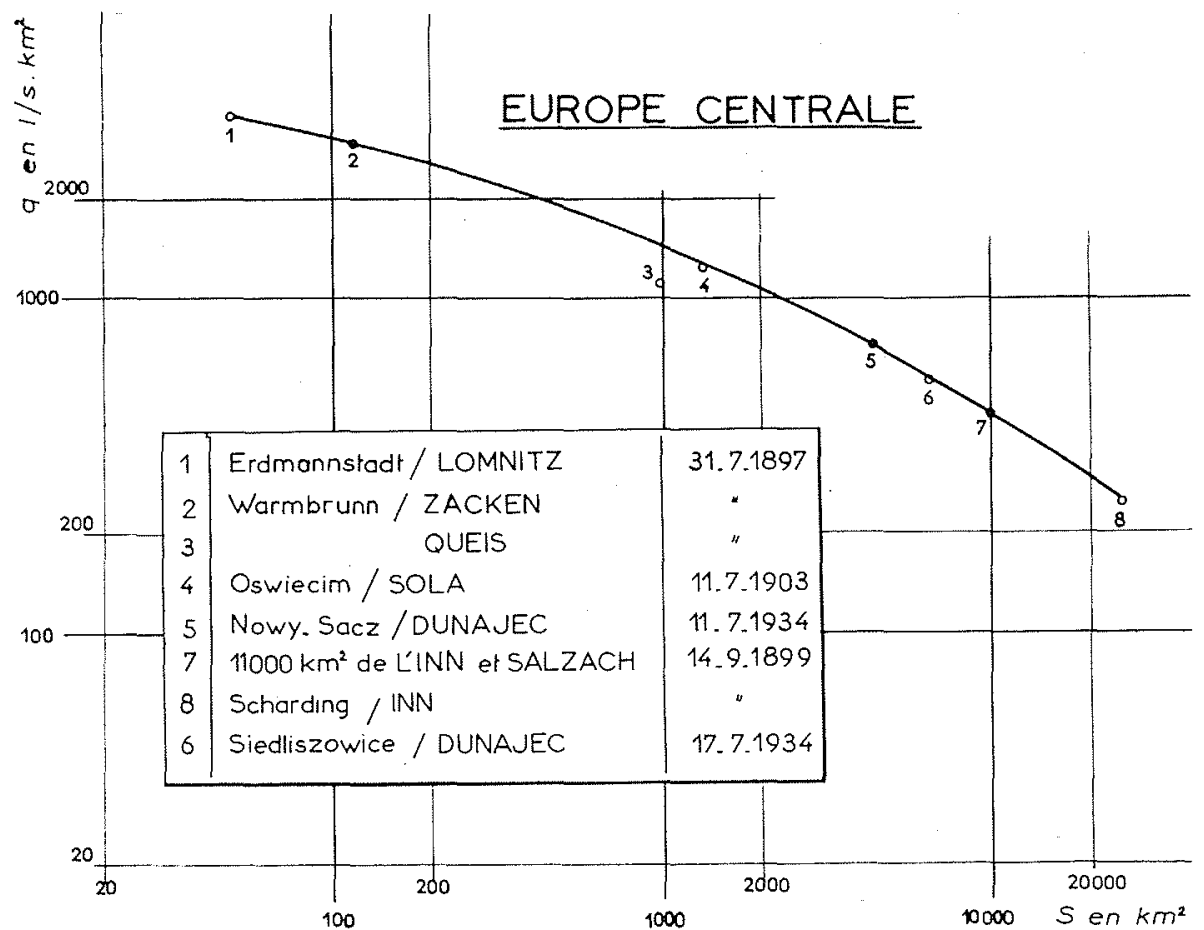

Pour la Californie du Nord, M. Pardé a manifesté une certaine inquiétude devant le débit maximal de l'Eel à Scottia survenu en 1955: à un bassin de $7950 \mathrm{~km}^{2}$ correspond un débit de $15320 \mathrm{~m}^{3} / \mathrm{s}$, débit qui, même réduit de 10 à $15 \%$, ne rivaliserait avec aucune crue observée pour une surface réceptrice de cet ordre dans les pays tempérés, même méditerranéens. Ainsi, si ce chiffre est exact, il a des chances de représenter une crue plus extraordinaire, plus rare que celle auxquelles correspondraient en général nos équations.

$2^{\circ}$ Pour le second type de courbes relatif aux trois grandes régions: Russie, Afrique noire, Amérique du Sud, nous avons repris une for-

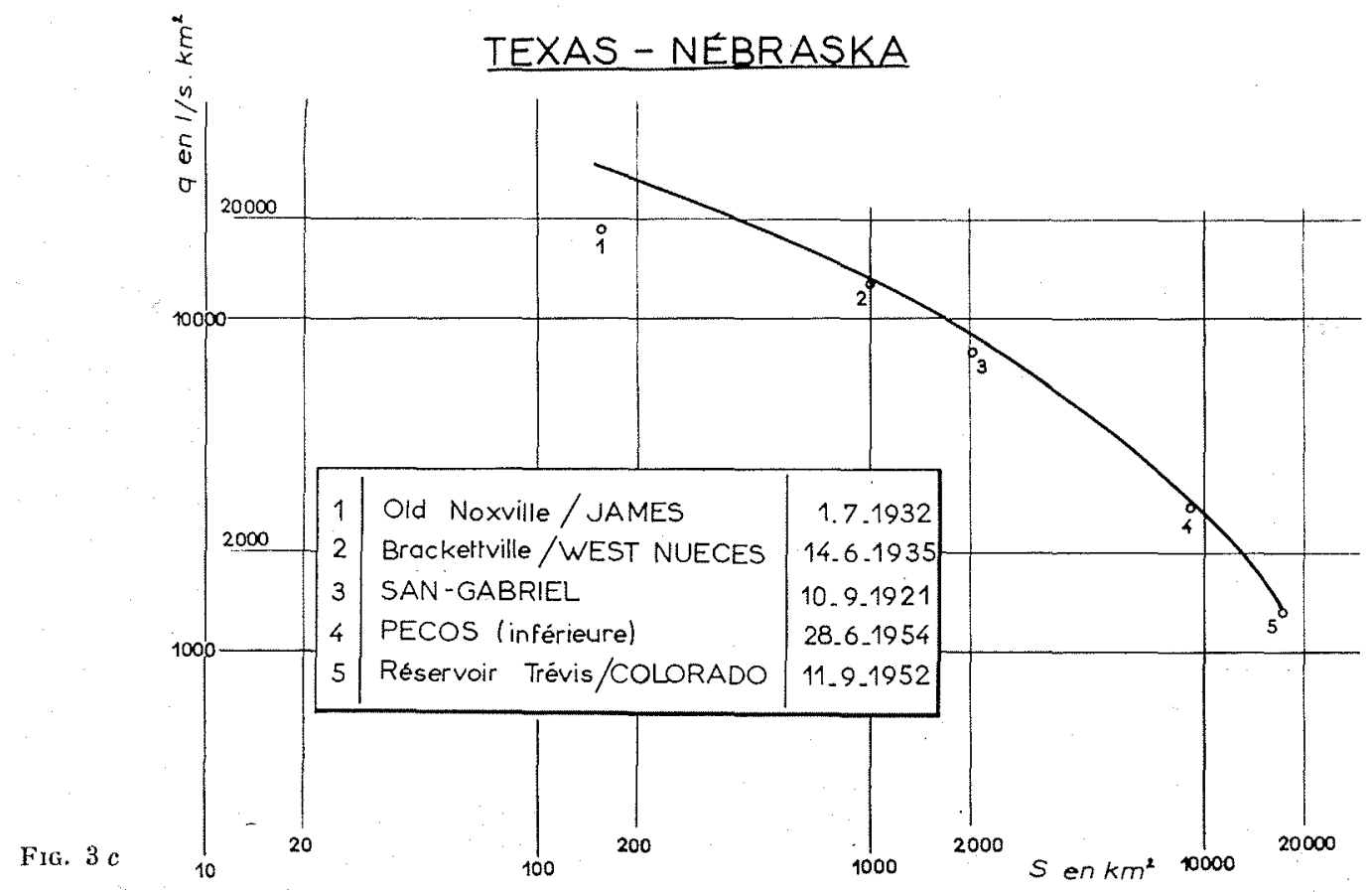




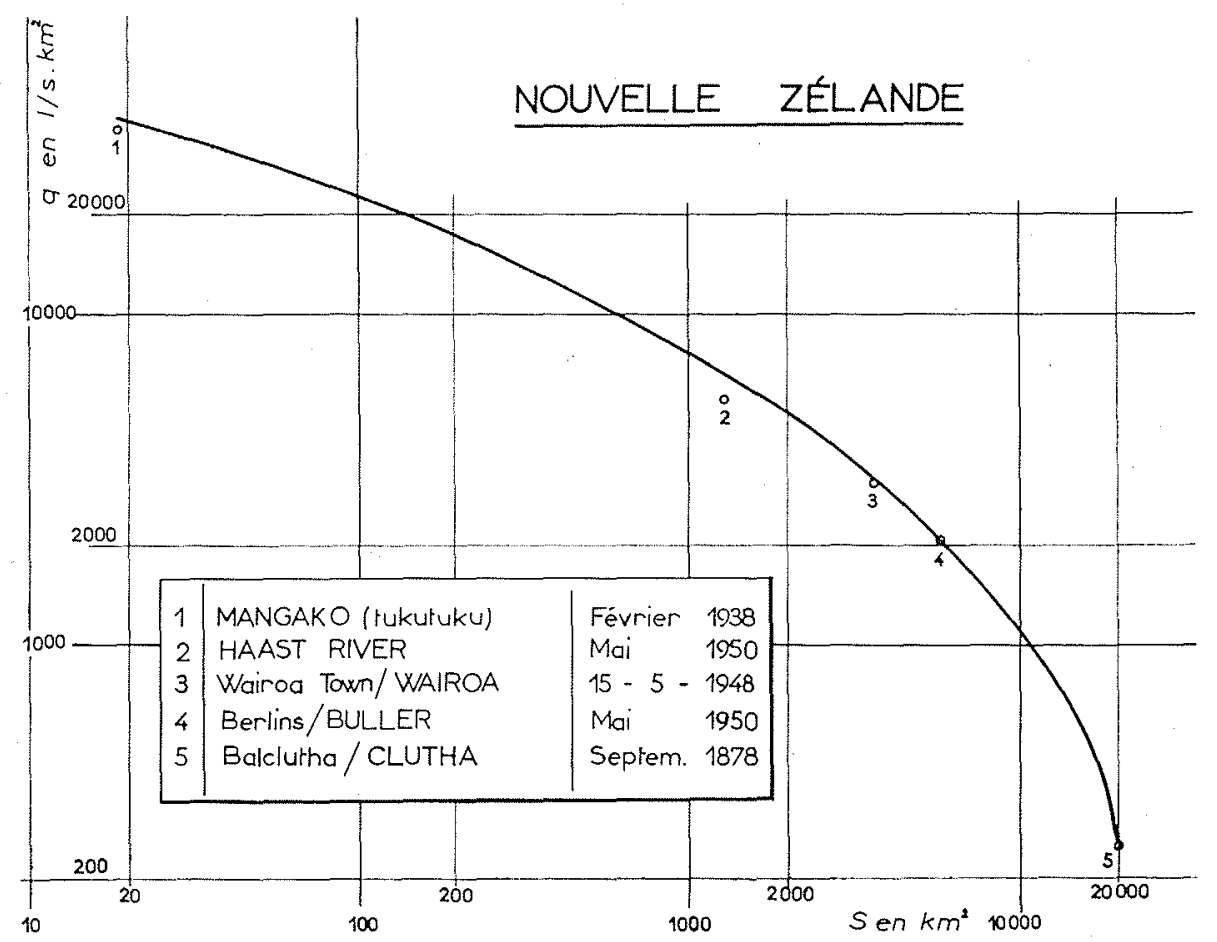

FIG. $3 d$

mule déjà exploitée, en particulier par les Italiens :

$$
q=\frac{\mathrm{A}}{\mathrm{B}+\mathrm{S}}+\mathrm{C}
$$

qui conduit à de très bons résultats (fig. 4). Le tableau ci-dessous précise la valeur des paramètres pour chacune des trois régions:

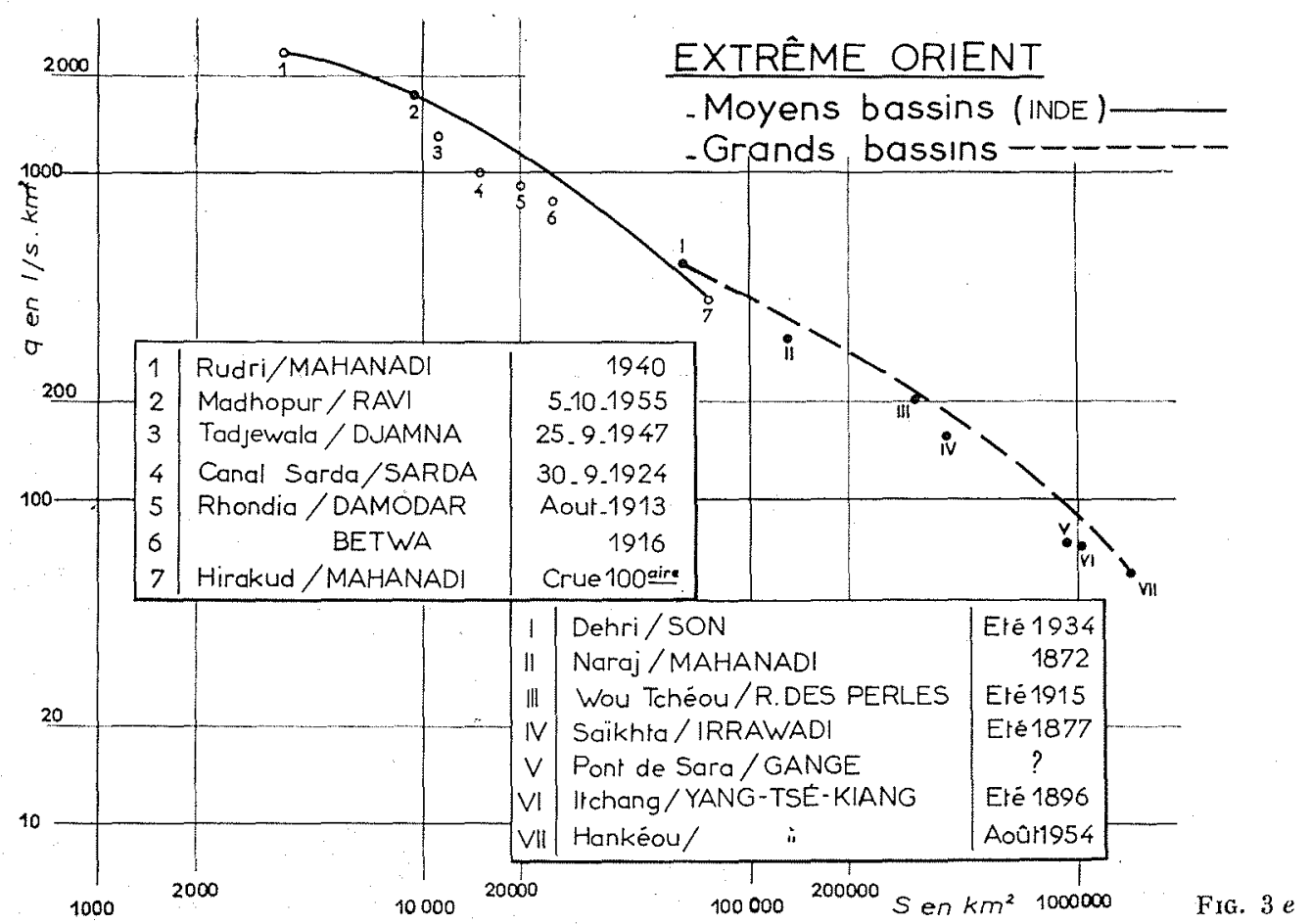


FIG. 4

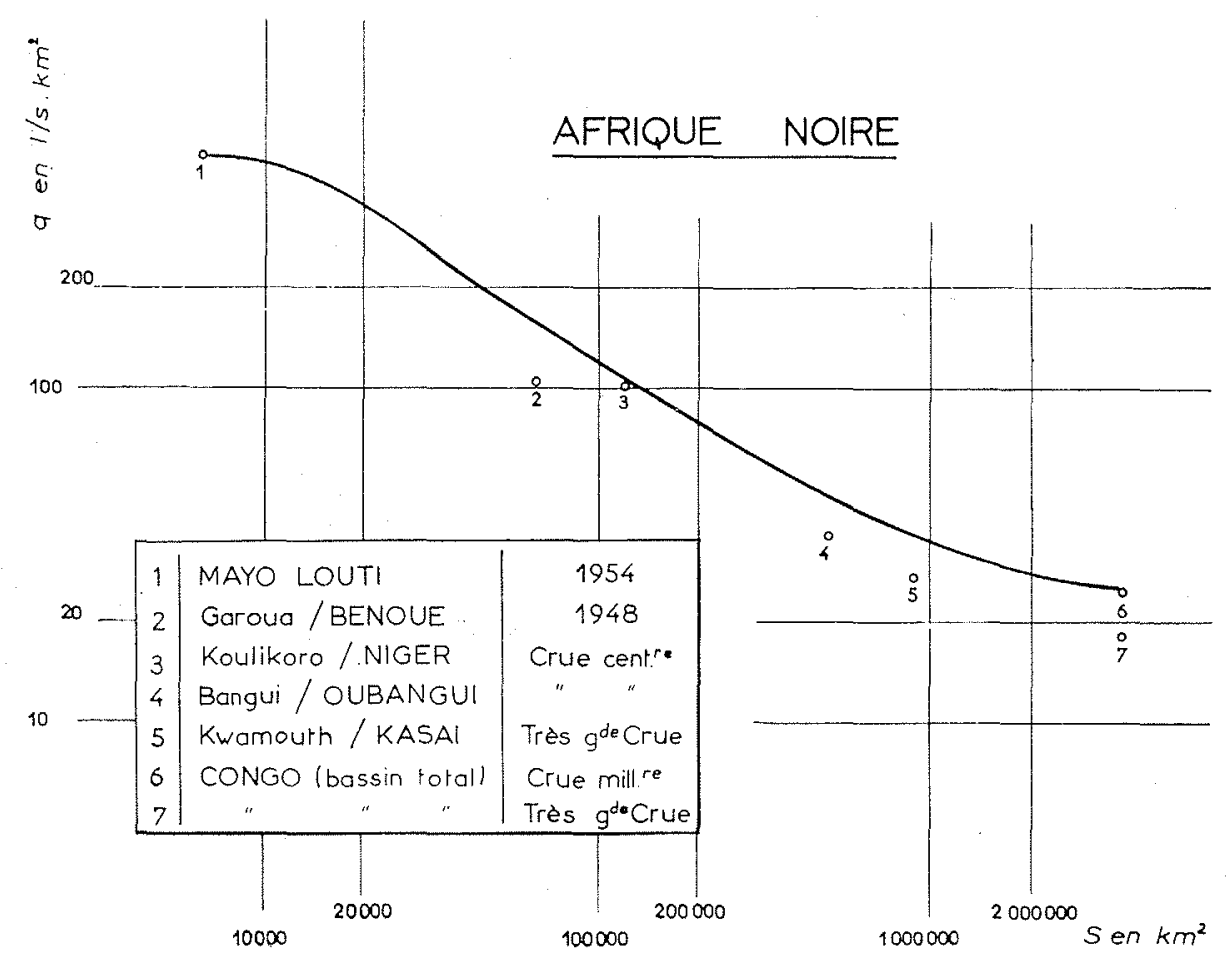

\begin{tabular}{|c|c|c|c|c|}
\hline " & $A$ & B & $\mathrm{C}$ & $S_{i}$ \\
\hline Afrique noire $\ldots \ldots \ldots \ldots \ldots \ldots$ & $10,6 \cdot 10^{6}$ & 15500 & 22 & $90000 \mathrm{~km}^{2}$ \\
\hline Amérique du Sud & $37,4.10^{6}$ & 34500 & 28 & $220000 \mathrm{~km}^{2}$ \\
\hline U.R.S.S. 1 & $28 \quad .10^{6}$ & 71900 & 37 & $240000 \mathrm{~km}^{2}$ \\
\hline U.R.S.S. 2 & $38 \quad .10^{6}$ & 187000 & 33 & $500000 \mathrm{~km}^{2}$ \\
\hline
\end{tabular}

(1) Deux courbes ont été établies pour la Russie. M. Pardé pense en effet que, dans la plus grande partie de cette région, les crues maximales pour 10000 et $100000 \mathrm{~km}^{2}$ environ ne dépassent point certains chiffres assez médiocres, en fonction desquels est tracée la courbe la plus basse. La courbe la plus haute, qui s'appuie sur les crues de l'Oka, la Belaïa, le Tom, correspondrait aux crues soviétiques possibles dans certaines régions privilégiées.

En quatrième colonne figurent les valeurs des surfaces qui correspondent au point d'inflexion de la courbe enveloppe ajustée en coordonnées logarithmiques:

$$
\mathrm{S}_{i}=\sqrt{\frac{\mathrm{AB}+\mathrm{CB}^{2}}{\mathrm{C}}}
$$

\section{CONCLUSION}

L'exploitation des résultats auxquels nous sommes parvenus reste, certes, un problème délicat. Pourtant, il ne nous semble pas ressentir une confiance déraisonnable en pensant que l'emploi de nos courbes donne des chiffres assez approchés en cas de crues exceptionnelles qui peuvent, dans les différentes régions, gonfler les rivières les plus redoutables. 
Avec plus d'audace, M. Pardé espère même que nous avons des chances sérieuses de représenter par nos courbes des débits maximaux de probabilités comprises, pour la plupart des stations, entre 0,006 et 0,003 .

Bien sûr, la détermination de la courbe enveloppe relative à une région donnée a nécessité un certain nombre d'interpolations. La région elle-même a été délimitée de façon plus ou moins grossière et lorsqu'on voudra se faire, à partir de nos courbes, une idée du débit maximal d'une rivière à une station donnée, on devra parfois prendre quelques précautions pour définir la région dans laquelle se situe le bassin considéré.

Il est évident, d'autre part, que dans la plupart des régions, les maximums de certaines rivières à certaines stations sont très inférieurs, parfois de $50 \%$, voire des $2 / 3$, au chiffre relevé sur la courbe enveloppe. Aussi est-il indispensable d'avoir sulfisamment de connaissances en hydrologie fluviale et de bon sens pour détecter les cours d'eau qui risquent de ne jamais avoir de débits supérieurs aux $2 / 3$, à la moitié ou au tiers des débits trouvés à partir de l'équation.
Par exemple, la Seine à Paris (maximum connu : $53-54 \mathrm{l} / \mathrm{s} . \mathrm{km}^{2}$ en 1910 ) n'arrivera peutêtre jamais à débiter le tiers du chiffre record observé ou à craindre pour la Loire et l'Allier réunis au Bec d'Allier (crue maximale observée : $225 \mathrm{I} / \mathrm{s} . \mathrm{km}^{2}$ en 1866). L'équation supérieure ne peut donc suffire à beaucoup près pour l'évaluation des très grandes crues de toutes les rivières françaises ou d'Europe centrale, par exemple. Mais elle peut quand même servir de base à cette évaluation.

Il faut en prendre son parti et étudier les causes des crues exceptionnelles, leurs facteurs de puissance ou de médiocrité et savoir utiliser force données comparatives pour trouver des débits maximaux records vraisemblables dans les différents bassins. Il peut d'ailleurs être beaucoup plus intéressant d'établir des équations supérieures valables pour des zones plus restreintes, certains grands bassins ou certaines zones géographiques homogènes à l'intérieur de grands ensembles, plutôt que de se laisser séduire par le charme de l'équation unique supposée valable pour toute une grande région.

\section{I S C USS I O N}

Président: M. de Rouvilue

M. le Président, avant d'ouvrir la discussion, remercie Mme LaRivalle qui a présenté cette communication et donne la parole à M. Pandé.

M. Pardé félicite Mme Larivalle pour son excellente communication. Le texte de celle-ci figurera en appendice du live «Sur la puissance des crues dans les diverses parties du monde» que l'Institut de Géographie de Saragosse va publier sous la signature de M. PAndÉ.

Ainsi que M. Pardé le rappelle, les courbes de Mme Laduvallze sont destinées a représenter non point les crues maximales jugées possibles ni celles de fréquence très rares, toutes les mèmes, calculees de manière à assurer l'homogénéité de tous les chiffres. Cette homogénéité est encore plus inaccessible que souhaitable. Mais Mme JAmivalle a voulu établir les courbes-enveloppes pour les plus grandes crues comnues. Les probabilités de cellesci sont de toute façon très faibles (peut-être de 0,006 à 0,003). Et ces courbes convexes ver's le haut, pour la plupart, en fonction de coordonnées logarithmiques ( $q$ en litres/seconde par $\mathrm{km}^{2}$, et $\mathrm{S}$ en $\mathrm{km}^{2}$ ) evitent les erreurs, dont certaines sont énormes, que comportail, pour des jcux élendus de surfaces réceptrices, l'emploi d'équations du type $\rho=A S^{n}$, avec un seul exposant pour le monde entier et un seul coefficient par région.

M. le Président pense que ces aperçus donneront à beancoup d'auditeurs le goût de consulter le livre de M. Pandé des sa parution.

M. Rodien voudrait souligner l'intérêt des courbesenveloppes dans les régions où les données sur les crues de faible fréquence sont rares : elles ont, par exemple, rendu de grands services à Madagascar et à l'île de la Réunion.
D'autre part, Ie point d'inflexion des courbes relatives à l'Afrique noire s'explique comme suit : les cours d'eall à gauche du point d'inflexion correspondent au régime tropical, les cours d'eau à droite de ce point, au régime équatorial, dont les crues sont moins violentes, plus prolongées et s'amortissent moins mpidement de l'amont vers l'aval. En fait, on a affaire a deux courbes simples tombantes, comme les autres, l'équatoriale étant beaucoup plus aplatie que la tropicale. Il est bien probable qu'il en est de même pour l'Amérique du Sud, le point d'inflexion résultant des crues de l'Amazone al régime équatorial, alors que les points voisins de laxe des ordonnées correspondent aux régimes tropicanx d'Amérique du sud.

Enfin, très sagement, l'auteur n'a pas compris dans son étude les petits bassins en dessous de quelques cencentaines de $\mathrm{km}^{2}$. On rencontrerait-la une tres grande hétérogénéité qui ne permettrait pas de tracer de telles courbes-enveloppes. Par exemple, pour l'étude du Kouilou, in calcul systematique des crues décennales donnait pour des bassins élémentaires de superficie standard $25 \mathrm{~km}^{2}$ : de $60 \mathrm{l} / \mathrm{s} . \mathrm{km}^{2}$ a $5500 \mathrm{l} / \mathrm{s} . \mathrm{km}^{2}$ suivant la nature du sol, alors que pour $10000 \mathrm{~km}^{2}$ les résultats étaient beaucoup plus homogènes et que, pour $57000 \mathrm{~km}^{2}$, le chiffre de $70 \mathrm{I} / \mathrm{s} . \mathrm{km}^{2}$, pour la crue décennale, était très compalable à celui de nombreux bassins de même surface de régime équatorial. On n'atteint donc un ensemble de compensations suffisantes qui ne rendent les bassins d'un même régime comparables, qu'au-delà d'une certaine superficic.

M. le Président approuve M. Rovier pour le fait qu'i! vaut mieux considérer du point de vue climatique une 
l'égion naturelle qu'un pays. C'est un très bel effort d'avoir essayé de mettre les phénomènes climatiques en courbes dans les régions homogènes. M. Ie Président en remercie Mme LarivallLe et $M$. Pardé qui se complètent l'un l'autre, Mme Larivarlee par ses connaissances mathématiques ef M. PARDÉ par son énorme documentation.

M. le Président remarque que ni le Nil, ni le Yang-TseKiang ne paraissent avoir été compris dans ces courbes

M. Pandé dit qu'il parle, dans son livre, beaucoup du Yan-Tse-Kiang et un peu du Nil *

M. le Président dit qu'il attend avec impatience la sortie du livre de M. PandÉ et remercie à nouveau l'auteur ainsi que Mme Larivallite

(*) Après la réunion, Mme Lanrvarure apporte la précision suivante :

Si le Nil n’a effectivement pas été pris en compte dans nos courbes, le Yang Tsé Yiang par contre $y$ figure en bonne place. Un des graphiques présentés en témoigne d'ailleurs : on peut constater en efret que la courbe-enveloppe relative aux crues d'Extrème-Orient (grands bassins) s'appuie en particulier sur la crue du Yang 'Tsé Kiang survenue en août 1954 à Hanliéou ( $\left.\mathrm{S}=1445000 \mathrm{~km}^{2}, q \cong 59 \mathrm{l} / \mathrm{s} \mathrm{km}^{2}\right)$. On remarquera de plus que le débit maximal relevé à Itchang durant l'été 1896 a été également pointé.

\title{
NOTRE FRONTISPICE
}

\author{
(Cf. p. 318)
}

\section{FIenri BOUASSE (1866-1953)}

Le "non-conformisme ) de la pensée d'Henri Bouasse, l'agressivité de ses célèbres préfaces, la longévité de son tempérament combatif, ont fait que sa mort n'a ell qu'un faible retentissement combatif, ont fait que sa mort n'a eu qu'un faible retentissenent
dans les milieux officiels francais. Aussi est-ce à une revue dans les milieux officiels français. Aussi est-ce à une revue
étrangère (Engineering du 18 décenbre 1953) que nous emprunétrangère (Engineering du 18 décenbre 1953 ) que nous emprun-
tons ces quelques notes qui mettent en relief $1^{\prime \prime i n f l u e n c e ~ d e ~ c e ~}$ tons ces quelques
maitre illustre $(*)$.

Physicien issu de l'Ecole Normale Supérieure, il était imprégné d'esprit praticue. Il le mettra d'ailleurs en exergue d'un cours de plysique qui constitue sans doute le plus important des onvrages synthétiques écrits par un seul auteur : "Bibliothèque scientifique de l'Ingénieur et du Physicien", à quoi il ajoute "Beaucoup de science, mais en vue des applications". Cette collection comporte aujourd'hui plus de 45 volumes in octavo (20 000 pages de texte, 900 pages de préface et près de 600 pages d'index).

La somme de travail représentée par cet ouvrage est considérable, surtout que l'auteur ne constilta d'autre source d'information que l'expérience et que la plupart des phénomènes décrits ont été reproduits par Bouasse lui-mêne ou ses assistants dans son laboratoire.

"L'une des raisons de mon impopularité dans les cercles officiels, a-t-il écrit, tient à ce ciue je me contente d'expriner dans le langage ordinaire, sans phraséologie oratoire, ce qui est au plus profond de la vérité, et de raisonner invariablement sur les exemples les plus banals. I a valeur éducative d'une science réside non pas dans ses conclusions qui peuvent toujours étre reside non pas dans ses conclusions qui penvent toujours etre controversees, mais dans la nature des preuves sur lesquelles elles sont fondes. Pour etre valable, un enseignement doit
rester le plus press possible des applications qui permettent de rester le plus pres possible des application
fixer les conditions de validité des lois. $)$

frxer les conditions de validite des lois. "
Les huit premiers volumes de la Bibliotheque scientifique renLes huit premiers volumes de la Bibliothèque scientifique renferment les fondements mathematiques utilises dans les volumes suivants; les six volumes suivants constituent un cours complet de meanique. Sult un volume traitant de l'elasticité et de la resistance des nateriaux, ou sont restmées les recherches personnelles de Bonasse. L ensemble des huit volumes suivants est consacré a l'étude des fluides. Les deux volumes de thermodyra mique parurent en 1913; le cours de magnétisne et d'électricite occupe trois volumes. L'optique ne compte pas moins de six volumes dont un sur l'optirue géométrique, un sur les instrument d'optique, un autre "Reproduction et vision des formes et des conleurs \%, deux enfin sur les interférences et la diffraction, écrits en collaboration avec l'abbé Carrière.

Les detx sections suivantes renfernent quatre volumes sur la cristallographie, l'étude des symétries et des propriétés optiques des nilieux cristallins et autant sur l'optique électronique, y compris les oscillations électriques, les ondes hertziennes, l'émission, la propagation et la réflexion de la lumière. Vien. nent ensuite sept volumes sur l'acoustique, sujet de prédilection nour Bouasse, fin nélontane et très bon pianiste.

En 1932, il entreprit une série de recherches expérimentales d'hydrodynamique, dont les résultats parurent entre 1933 et
1939 successivement dans les Annales de physique, les Annales 1939 successivement dans les Annales de physique, les Annales
de la Faculté des sciences de loUniversité de Toulonse et les

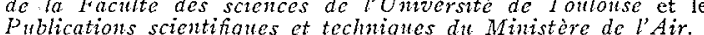
Publications scientifiques et techniques du Ministere de l'Air. Bouasse fut probablement 'un des derniers representants de était the joie, et non une fastidiense interruption dans la recherche. Il reçut peu de titres de reconnaissance officielle et n'en sollicita aucun, mais l'influence exercée par son cuvre
monumentale, la Bibliothégue scientifique de l'Ingétiewr et du monumentale, la Bibliothèque scientifique de l'Ingénien

(*) La Houille Blanche a également publié une notice biograi)hique sur Henri Bouasse dans son numéro 3, de juin 1954 , page 425

\section{Henri BOUASSE (1866-1953)} The "non-conformist" ontlook of Henri Bonasse, the aggressive
tone of the frefaces to his many works, and the indomitable fighting spirit he showed right to to the very last all help to fighting spirt he showed right th to the very last all help to
explain why his death passed practically unnoticed in French explain why his death passed practically unnoticed in French
official circles. The fact, need hardly come as a surprise, thereofficial circles. The fact, need hardly come as a surprise, therefore, that information has tad to be borrowed from a foreign publication. (Engineering-18 Decemoer 1953) in compiling

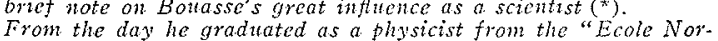
From the day he graduated as a physicist from the "Ecole Northale Superieure", to the end of his career, Honri Bouasse never
losi his essentially practical outlook, which is probably best lost his essentially practical outlook, which is probably best
stmmed up by the motto appearing on the titlo page of every summed up by the motto appearing on the titlo page of every
volume of his "Bibliotheque Scientifique de l'Ingenieur et du volme of his "Bibliothèue Scientifique de l'Ingenieur et du Physicien" with the words : "Beanconp de science, mais cn vile
des applications." This collection of auorhs, which is the most comprehensive ever written by a single anthor, foday includes more than 45 oclavo volumes containing 20,000 pages of text, some 900 pages of preface, and nearly 600 pages of index. The magnitude of this 1tndertaking is quite staggering, especially as the author solely relied on experience for his information; moreover, most of the experiments described atere carried out in Bouasse's own laboratory, either by the anthor himself or by his assistants.

"One of the reasons of my unpopularity", he once zurote, "is that I am content to express the most profound truths in simple everyday langnage devoid of eloquent phraseology, and to reason invariably on the most commonplace of examples in their simits conclusions, which are alatays subject to controversy, but in the nature of the proofs whereby they are established. If teaching is to be of any value it must remain as closely associated as possible with the applications rohich serve to determine the conditions under when scicitific wans are walid."

The first cight volumes of the Bibliothèque Scientifique contain the mathematical foundations for the succeding volumes; the nexi six are a complete course in mechanics, followed by a volume on elasticity and strength of materials which is a summary of Bonasse's own research. Eight volumes are given over to a study of fluid states, two deal with thermodynamics, and three more cover a course on naynetism and electricity. Si. volumes on optics include one on optical geometry, one on optical instruments, one entitled "Reproduction et vision des formes et des conlurs"; two compiled in collaboration with Abbé Carriere deal with. interference and diffraction.

The next two sections include four volumes on crystallography, a sludy of the symmetry and optics of crystaline media, four volumes on electro-optics, including electrical oscillations, Hertsian ataves, and the emission, propagation and reflection of light. The last seven volumes are concerned with acoustics, a subject to which Bouasse, an accomplished pianist and a keent amateu musician, wers particularly devoted.

In 1932, Bouasse undertook a series of experimental investiga tions in hydrodynamics, the results of which were published betwecn 1933 and 1939 successively in Annales de Plysique, Annales de la Faculté des Sciences de l'Universite de Toulouse, and Publications Scientifiques at Techniques du Ministère de I'Air.

Henri Bouasse was probably one of the last of those professors Henri Bouasse was probably ane of the last of those professors
of the "old school" to whom teaching was a joy and not merely a of the "old school" to whom teaching was a joy and not merely a
tiresome intermption to rescarch. He received little official recognition and asked for none. Nonetheless, the infuence of his truly monumental Bibliothè uue Scientifique de I'Ingénieur of his truly montmental Bibliothèue Scientifique de l'Ingenieut
et du Physicien is not only frmly established but rill remain so et du Physicien is not only
for many a year to come.

(*) A biographical note on Henri Bouasse also appeared in La Howille Blanche No. 3, June 1954, p. 425. 